\title{
Una política pública de la mujer con perspectiva de género
}

Olga Lucía Obando Salazar 



\section{Una política pública de la mujer con perspectiva de género}

Olga Lucía Obando Salazar

Recibido: Septiembre 2006

Aceptado: Octubre 2006
* Dr. Phil. Olga Lucía Obando Salazar. Grupo GEMA - Línea Psicología Política. Instituto de Psicología. Universidad del Valle 


\title{
UNA POLÍTICA PÚBLICA DE LA MUJER CON PERSPECTIVA DE GÉNERO
}

\author{
Olga Lucía Obando Salazar
}

\begin{abstract}
RESUMEN:
Revisar aspectos referentes a la perspectiva de género en la planeación, diseño, construcción, implementación y evaluación de políticas públicas de mujeres, como un proceso local y regional, al interior de otros más amplios como los de Globalismo, Gobernabilidad y Territorio constituye el eje central de la ponencia. El texto se estructura en tres partes: en la primera, se trabajan algunas inquietudes que se generan en la lectura crítica de los documentos: "Política de mujeres constructoras de paz y desarrollo" y "Lineamientos de política de equidad de Género para las mujeres de diferentes sectores y grupos étnicos del Valle del Cauca". Estas inquietudes se refieren a la utilización implícita y explicita que en los textos se hace de los conceptos de: Política pública, Mujer y Género. La segunda parte hace referencia al papel de los agentes que participan en la construcción, gestión e implementación de la política pública de la mujer. Y en un tercer aparte se resume la situación del actual desarrollo de una política pública de la mujer a nivel del departamento del Valle del Cauca.
\end{abstract}

\section{PALABRAS CLAVE:}

género, políticas públicas de mujer, participación política.

\section{ABSTRACT:}

The central issue of this presentation is to review aspects refering to the perspective of Gender in the planning, design, construction, implementation and evaluation of public policies of women, as a local and regional process, inside other wider processes such as Globalism, Governability and Territory. The text is structured in three parts: in the first one are treated some discomforts that are generated in the critical reading of the documents "Women constructors of peace and developments policy" and "Features of policy of fairness of gender for the women of different sectors and ethnic groups from the Valle del Cauca". These restlessness talk about the implicit and explicit use made in the text of the concepts of Public Policy, Woman and Gender. The second part refers to the role of the agents that participate in the construction, managment and implementation of woman's public policy. And in a third part the situation of the present development of a woman's public policy in a departmental level (Valle del Cauca) is resumed.

\section{KEY WORDS:}

Gender, woman's public policies, political participation. 


\section{Antecedentes}

Revisar aspectos referentes a la perspectiva de género en la planeación, diseño, construcción, implementación y evaluación de políticas públicas de mujeres, como un proceso local y regional, al interior de otros más amplios como los de Globalismo, Gobernabilidad y Territorio constituye el eje central de la ponencia.

Un primer aspecto se refiere a la importancia de ubicar las propuestas locales de construcción de una política pública de mujer dentro de un contexto global. Tres características de la Globalización: la deslocalización, la movilidad y la interconectivilidad afectan la planeación, el diseño, la construcción y la implementación de políticas públicas de mujeres. La aceptación del ejercicio de una democracia política global regida por principios de equidad y dinámicas de participación incide en aspectos referentes a los asuntos de género a nivel local. Es así como un acumulado de leyes, resoluciones y decretos, firmados por entes internacionales con reconocimiento global ${ }^{1}$ jalonan reformas al interior de las cartas constituciones nacionales y un desarrollo de marcos jurídicos y de gestión pública que son operacionalizados como garantes del cumplimiento de ciertos perfiles del ciudadano global, un ciudadano democrático, equitativo, participativo. Estas innovaciones en la gestión de políticas públicas son valo- radas como un aporte de las naciones que se proponen el poder llegar a ser aceptadas como parte integrante de ese mundo globalizado. Es interesante revisar los efectos de las tendencias globalizadas de organizar los asuntos de las políticas públicas y su incidencia en la Gobernabilidad Local y Regional en naciones como Colombia.

Si entendemos por gobernabilidad: la capacidad que tiene un Estado de llevar a cabo unos objetivos programáticos y lograr metas, en el caso especifico, referido a la construcción de una política pública de la mujer, que responda a las exigencias del mundo globalizado, se le plantea a la Gobernabilidad local y regional un primer problema y éste se refiere al legislativo. Su apariencia es en la forma de un problema técnico, en tanto existe una falta de preparación de muchos de los agentes legislativos sobre la realidad implicada en la norma. Autoras como Hurtado señalan, cómo la aprobación de muchas Leyes (por ejemplo: la Ley de cuotas políticas para mujeres, como uno de los casos revisado por la autora) "surgen más como un caso de coyuntura política que de voluntad democrática" (Hurtado, 2005, p 105, citado en (Obando, 2006d, p. 2). Incorporar territorios y colectivos a una lógica del sistema económico mundial, el de la Globalidad, con sus inherentes contradicciones de principios políticos democráticos $\mathrm{y}$ modelos de desarrollo económico que Algunas de las instituciones internacionales citadas en el marco jurídico del documen-
to de ley de mujer justicia y paz. 
fortalecen la exclusión social, implica el hacer rupturas con marcos simbólicos existentes. González plantea, que "las desigualdades sociales y las distintas formas de exclusión social aumentan día a día como consecuencia de la implantación de modelos de desarrollo que convierten en hegemónicas las fuerzas del mercado y reducen al mínimo el papel regulador del Estado" (González, 1999, p.2). El territorio se entiende como la memoria, la lógica que le compete a cierto grupo. Para el caso referido a una política pública de mujer con equidad, el problema radica en el hecho de que en la mayoría de las veces esta innovación a nivel legislativo no se encuentra acompañada de una oferta por parte del Estado de nuevos marcos simbólicos para interpretar las transformaciones que implican la construcción, evaluación y resignificación, de una nueva política pública de mujeres de inclusión social y equidad. Una política que se superponga al estado de exclusión impuesto a las mujeres. Aquí la exclusión se entiende como "...la imposibilidad de asumir la ciudadanía, entendida como la capacidad de ejercer los derechos políticos, sociales, culturales y económicos." (González, p.3).

Construir la política pública de mujer para el Valle del Cauca, desde una perspectiva democrática, de equidad y de género debe conllevar a abrir canales de diálogo entre las mujeres, y de éstas con las entidades del sector público y privado, para establecer compromisos y definir responsabilida- des frente a la situación y el papel de este sector de la población a nivel local.

\section{Algunas ambigüedades en el manejo de los conceptos de Política pública, Mujer y Género}

Contestar a la pregunta sobre ¿Qué es una política pública de mujer? implica tomar posición frente al concepto de política pública y frente a la razón de ser de ésta, como una política pública de género.

Es quizás el reconocimiento de la existencia de diferencias que se tornan en desigualdades, lo que permite la construcción de medidas destinadas a grupos especiales, que buscan remediar por la vía oficial y legal las inequidades (Obando 2006d, p.1), es decir la construcción de políticas públicas específicas a grupos y problemas. El reconocimiento de la existencia de un sistema de inequidades adscrito a la pertenencia de género es lo amerita la construcción de una política pública de mujer con perspectiva de género.

Cuando se asume la tarea de participar en un proceso de construcción de una política pública, es necesario tomar consciencia de que existen varias vías para resignificar el concepto de política pública y cada una de ellas se apoya en una postura teórica que plantea un tipo de relación entre el Estado y la política pública. Roth Deubel (2004), identifica tres grandes posturas teóricas "las teorías centra- 
das en la sociedad, las centradas en el Estado y las teorías mixtas o intermedias." (Roth Deubel 2004, p.29).

En analogía con Roth Deubel el significado que adquiere la determinación de hacer una política pública de mujer centrada en la sociedad se refiere al hecho de que en ella el Estado es definido como una variable dependiente, es decir que en la construcción de esta política pública se minimiza la capacidad y el impacto que pueden tener las instituciones públicas, sus agentes y los valores, sobre las elecciones hechas en materia de dicha política. Desde esta postura le correspondería a las mujeres como individuos o grupos determinar las políticas públicas ha desarrollarse. Esta política pública aparecería como el resultado de las presiones sociales realizadas por las mujeres (Roth Deubel 2004, p.29).

En el desarrollo de una política pública de mujer centrada en el Estado se le otorgaría a los Individuos y grupos que conforman el Estado la potestad para determinar las elecciones que en materia de políticas públicas fuesen pertinentes. La construcción de una política pública como acción pública aparece en este enfoque como "el resultado de elecciones realizadas por los decidores políticos y por los altos funcionarios públicos", de esta forma se logra neutralizar la influencia de "los actores situados en el entorno social, económico, politico e internacional del estado para explicar las elecciones de políticas públicas". (Roth Deubel 2004, p.30) y minimizar la influencia de los cambios en las decisiones que el Estado tome con relación a esa política. En último se trataría de una política pública de mujer construida en base a la racionalidad de los burócratas y políticos. Propuestas como: la construcción de una Política pública de Mujer y Familia, impulsadas desde los grupos de poder con posturas argumentativas tradicionales o religiosas testimonian dicha tendencia.

Quizás el enfoque más acorde para el desarrollo de una Política Pública de Mujer con perspectiva de Género en la realidad departamental del Valle del Cauca, es el de una teoría mixta o intermedia, la cual como lo plantea Roth Deubel,

"intentan tomar en consideración con todas las distinciones y variaciones que existen al interior de ellos tanto factores internos (centrados en el Estado) como externos (centrados en la sociedad) en su explicación de la acción pública y de las politicas públicas. (Roth Deubel 2004, p.31)

Los itinerarios de los procesos de construcción de políticas públicas de la mujer en Colombia a nivel departamental y municipal se apoyan en procesos de gobierno central. Es de allí que la existencia de la CONSEJERÍA PRESIDENCIALPARALAEQUIDAD DE LA MUJER ${ }^{2}$ como entidad rectora de las políticas para mujeres juegue un

2 Constituida bajo el decreto 519 del 5 de marzo del 2003 de presidencia de la República de Colombia. 
papel directriz. Es misión de esta consejería:

"Diseñar, promover, coordinar e implementar una politica para mujeres adultas, jóvenes y niñas, que contribuya al logro de relaciones de equidad y de igualdad de oportunidades entre mujeres y hombres, eleve la calidad de vida de las mujeres, el respeto de los derechos humanos, la participación ciudadana, e impulse el fortalecimiento de los procesos organizativos y de las organizaciones de mujeres. (Presidencia de la República, 2003, p.14)

Esta consejería tiene como objetivos generales la estructuración de la política nacional para las mujeres; la transversalización de esta política en la Administración pública y el Fortalecimiento de la institucionalización de la política. (Presidencia de la República, 2003, p.14)

\section{Los principios de género en la política pública de mujer}

Una mirada ingenua a la misión y los objetivos de la Consejería Presidencial para la equidad de la mujer reportaría una analogía con algunos de los principios fundamentales EMANCIPACIÓN, PARTICIPACIÓN, EMPODERAMIENTO, del discurso teórico y del quehacer práctico de los trabajos de la mayoría de las propuestas de género y en especial las lideradas por los movimientos de mujeres, particularmente por grupos de mujeres feministas. Estas propuestas plantean en sus metas una crítica al patriarcado y un fortalecimiento de la emancipación de mujeres. (Obando 2006b, p.12)

A pesar de que la propuesta de la Consejería expone lineamientos participativos y autogestionarios es necesario mantener la atención en las rutinas que la gestión de una política pública de la mujer a nivel local implica, sobre todo la tendencia del cuerpo de representación estatal a asumir dicha tarea como la propia de un ente burocrático, lo cual puede constituirse en el mayor obstáculo para el logro de sus objetivos y misión. Como lo plantea Campos, el Estado funciona como un operador de instrumentos y estrategias "las politicas públicas son instrumentos con los que el Estado pretende lograr ciertas metas a fin de lograr el bienestar de las sociedad; en éstas la participación de la sociedad se da de manera aislada y sólo en el caso en que los grupos tengan la información y los contactos necesarios para participar en éstas." (Campos, 2003, 86).

Si aceptamos que es menester construir las políticas públicas se hace necesario determinar el grado de contradicción o inconsistencia entre un discurso participativo y el ejercicio de un hacer burocrático.

El riesgo más grande que se corre cuando la construcción de la política pública se ejecuta como labor burocrática de operación de un instrumento u una estrategia radica en el hecho, de que el desempeño de esta tarea no implique (en obligatoriedad) una identificación, ni un sentimiento de pertenencia con el grupo y la causa a la cual va dirigida la propuesta. 
Ese vacío de involucramiento o implicación(Mies 1984, Palazón 1993, Obando 2006a) con la situación del grupo y el problema al cual esa política pública intenta responder, limita a ese Ente para reconocer la diversidad y multiplicidad de intereses, valores, necesidades, sueños y formas de resistencia que amarran y desamarran a los sujetos sobre los cuales recae dicha política, para el caso las mujeres.

Uno de los retos que asumen quienes se proponen la tarea de construir de forma cooperada una política pública de mujeres con perspectiva de género sea esta dentro de un modelo de participación cogestiva o de participación autogestiva (Campos 2003, p.86) es el poder acercarse a comprender qué es lo que se comparten los sujetos de este grupo en su diversidad: ¿La condición de pertenencia de género? ¿La condición de discriminación o exclusión basada en el sexo? (Htung, 2005), ¿La disposición a asumir una lucha por la equidad y la emancipación?

Al mismo tiempo esta falta de involucramiento o implicación puede desencadenar un Inter-juego de poder entre los intereses propios del sujeto político y social que asume la labor burocrática y los intereses del grupo meta. En la opinión de Foucault (1993), se hace necesario revisar el discurso de la política, en tanto ésta debe ser contemplada como portadora de un discurso de poder, que posee en sí mismo la facultad de empoderar o desempoderar a los sujetos a los cuales esta dirigido, LAS MUJERES, para el caso de la política pública de la mujer. En la formulación de políticas con enfoque de género se debe tener claro que no existe un componente de neutralidad ni el Estado ni en las políticas son neutrales, en tato éstas proyectan y reproducen las normas, los valores y tendencias vigentes de la sociedad en las cuales emergen (Silveira, 2000), incluso las percepciones sobre las pertenencias de género.

Una de las salidas a ese problema es quizás el abrir espacios de discusión a nivel de todas las esferas sobre las contradicciones existentes entre los fundamentos emacipatorios misionales y algunos de los objetivos específicos de la propuesta de la Consejería, como es por ejemplo el objetivo de "Diseñar, proponer y poner en marcha una política para las mujeres-adultas mayores, adultas, jóvenes y niñas- que propenda por su desarrollo integral, por su equidad de género y por un cambio cultural que valore su aporte al desarrollo", (Presidencia de la República, 2003, p.14). La contradicción radica en el hecho de que le es adjudicada a la Instancia la "Consejería" una tarea que le corresponde a las mujeres como agentes con capacidad de construir propuestas desde su lugar de implicación (Mies 1984, Obando 2002). Es decir, se le niega su capacidad autogestionaria (Arango 2006, Zúñiga 1996, Obando 2006a) lo que conlleva tal vez de manera involuntaria a desencadenar procesos en los cuales se construye desde este Ente institucional a las muje- 
res como meras consumidoras de los paquetes de políticas públicas, construidos por terceros, despojándolas del ejercicio de sus capacidades de sujetos políticos, reflexivos de su propia realidad social, emocional y cognitiva (Martin-Baro 1994; Fernández Christliebt 1987; Obando, 2002, 2006a). En una propuesta emancipadora y de género la participación de las mujeres debe superar el carácter de consumo, el lugar de convidadas de piedra, la construcción de esta política debe ser percibida como un proceso de cambio colectivo en el cual se vinculan las mujeres como sujetos afectados por la problemática, como lo plantea Torres Salcido (2003), deben ser políticas publicas de participación social. En estas políticas se parte del supuesto de que existe un público interesado, en las políticas, en los programas de gobierno y en las instituciones. Interesado en su diseño, implementación evaluación y rediseño. Una noción de lo público que supone "una ciudadanía participativa e informada que tiene espacios de discusión racional. La condición del ciudadano seria la esfera ideal" (Torres Salcido, 2003, 45).

Al respecto es de rescatar el primer punto del Acuerdo Social entre la Gobernación del Valle del Cauca y la Confluencia Departamental de Procesos Organizativos de Mujeres del Valle del Cauca, en el cual se propuso:

"Formular e implementar una política pública departamental de reconocimiento e inclusión social desde y para las mujeres, tendiente a fortalecer sus niveles de organización y articulación, su empoderamiento personal y colectivo, su acceso a los derechos, a las instancias de toma de decisiones y a lograr los cambios culturales necesarios para vivir en una sociedad equitativa" (Gobernación del Valle del Cauca, Acuerdo Social, 2004, p.3),

Quiero subrayar en este apartado, el "desde y para las mujeres".

Tanto los principios que rigen la Consejería presidencial de la mujer, como los que rigen la Política Nacional de Mujeres 2003-2006 "equidad, igualdad, participación, respeto a los derechos de las mujeres, respeto al derecho internacional humanitario, autonomía, respeto a la diversidad, descentralización" (Presidencia de la república, 2005, p.18-22), poseen un carácter emancipador, sin embargo son múltiples los ejemplos que muestran cómo su implementación plantea exigencias que superan los buenos propósitos expuestos en leyes, decretos y cartas constitucionales. Un ejemplo patente lo presentan las Autoras Magdalena León Y Jimena Holgín (2005), en el artículo "La cuota sola no basta" en donde se elabora un análisis crítico sobre el estado de cumplimiento de la Ley 581 del 2000 o Ley de cuotas para la administración pública y también lo plantea Angélica Bernal Olarte (2006), en el estudio "Coyuntura política en Colombia" cuando muestra el retroceso de la presencia de mujeres en las instancias del Gobierno como el Congreso, Senado y Cámara entre otros. 
A pesar de la existencia desde 1984, a nivel nacional ${ }^{3} \mathrm{y}_{\text {departamental }}{ }^{4}$ de un grupo de políticas para la mujer es innegable que en la vida privada, social y política la situación continúa sin muchos cambios. En Colombia persisten las relaciones inequitativas, es decir, "las reformas legales no han sido suficientes para alcanzar la igualdad de oportunidades en el acceso a la distribución del poder y la toma de decisiones, a la salud, educación, vivienda, ingresos" (Centro de Estudios de Género, 2006a, p.10-11), reafirmándose la faltas de preparación tanto de los agentes del legislativo como de los individuos y grupos sociales para sumir la realidad implicada en la norma de la equidad, como se planteo anteriormente. Se considera que a la par que se construyen las propuestas discursivas de políticas públicas para la mujer, es necesario trabajar en la construcción de marcos lógicos que permitan esclarecer la viabilidad de estos propósitos, agendas que permitan aclarar cuales procedimientos, actividades, mecanismos de análisis y evaluación de procesos favorecen el logro de objetivos específicos como los planteados en el documento de
Política de Mujeres Constructoras de Paz y Desarrollo:

- "Estimular los procesos democráticos de organización, participación y movilización de las mujeres en el ejercicio del poder y la toma de decisiones en los procesos políticos, económicos, culturales y sociales.

- Impulsar y fortalecer procesos de empoderamiento de las mujeres y apoyar estrategias de incidencia local, regional y nacional.

- Medir los impactos de los resultados esperados de la política de equidad para las mujeres y de las normas compromisos y acuerdos internacionales sobre el tema.

- Promover y garantizar escenarios que fortalezcan la implantación de la politica de equidad para la mujer y la responsabilidad que tienen las entidades territoriales en esta labor." (Presidencia de la República, 2003, p.15).

En este sentido se trata de trabajar en la construcción de una política pública de la mujer amarrada al desarrollo de una política social. Gonzáléz, citando al Foro Nacional por Colombia, define la política social como:

" el conjunto de orientaciones estratégicas, construidas a través de procesos

3 Política para la Mujer campesina 1984; Política integral para las mujeres 1992; Política Salud para las mujeres, Mujeres para la salud 1992; Política para el desarrollo de la mujer rural 1994; Política EPAM, política de equidad y participación de la mujer 1994; Avance y ajuste de la política de Equidad y participación de la mujer 1997; Política Nacional de salud sexual y reproductiva 2003; Política nacional Mujeres constructoras de paz y desarrollo 2003.

4 Política departamental para la mujer 1993; Política de atención a la mujer campesina e indígena 1993; Política de igualdad, equidad y participación para la mujer caleña 1995; Decreto 1677 de octubre 4 de 1996 un compromiso político para el logro de la igualdad, equidad y participación de la mujer de santiago de Cali. 
de diálogo entre el gobierno municipaly los distintos actores sociales, económicos y políticos, locales y extra-locales, dirigidas a garantizar la igualdad de oportunidades para todos en el ejercicio de sus derechos, en particular de los derechos sociales, asegurar los medios para el mejoramiento de la calidad de vida de la población, especialmente de los sectores excluidos y vulnerables y construir un capital social que sirva de fundamento a la convivencia pacífica." (González, 1999, p.3)

En el documento del Acuerdo Social se deja leer que tanto la Gobernación del Valle del Cauca como la Confluencia Departamental de Organizaciones de Mujeres asumen en buena parte este menester, es decir la responsabilidad por el diseño, construcción, implementación, evaluación y resignificación de una política pública de mujer con perspectiva de género, que combina los intereses de la política pública con los de una política social de mujeres. Dentro de los puntos acordados se asume que:

3. La Confluencia Departamental de Procesos Organizativos de Mujeres, generará propuestas, concretará y priorizará y apoyará programas y proyectos gubernamentales y no gubernamentales, generará opiniones y análisis, avalará iniciativas para la búsqueda de financiaciones y hará veeduría al desarrollo de políticas para el acceso de las mujeres en dichos programas.

4. la Gobernación a través de sus secretarías y demás dependencias, diseñará e implementará de manera concertada con la confluencia departamental de procesos organizativos de mujeres las politicas, planes, programas y proyectos que posibiliten el pleno desarrollo de la ciudadania femenina ...

5. La Gobernación incluirá concertadamente en el Presupuesto Departamental de los años 2005, 2006 y 2007, las partidas presupuestales para garantizar la implementación de las iniciativas que surjan del punto anterior. (Gobernación del Valle del Cauca, 2004, p.3)

Estos puntos del Acuerdo Social dan respuesta a algunas preocupaciones planteadas tanto por representantes del trabajo teórico y de base con mujeres, como por las mujeres mismas, en relación con la formulación de iniciativas sostenibles y permanentes que favorezcan el ejercicio activo y responsable de ciudadanía como mujeres, fortalezcan sus potencialidades para la exigibilidad de la garantía de sus derechos y les permita un vínculo a participar en procesos de reflexión y debate de asuntos de interés colectivo de género, así mismo como en la planificación, ejecución y evaluación de los programas y proyectos dirigidos a las mujeres. El proceso de construcción de una política social para mujeres que acompañe el proceso de política pública se erigiría como “...un instrumento trascendental para enfrentar los acuciantes problemas y como una forma eficaz para reorientar el desarrollo y mejorar la gestión pública." (González, 1999, p.1). La prioridad de la gestión que incluye la política social puede llegar a constituirse en un eje articulador del conjunto de políticas publicas municipales (Velásquez 2003) referidos a la mujer. 
Como grupo o individuo que participa en ese proceso de construcción de una política pública y una política social de mujer hay que estar atenta al hecho de que se mantiene una brecha entre el pensar y el hacer, como lo plantea Gonzáléz:

"en el caso de Colombia la política social ha estado implícita en los diferentes Planes de Desarrollo. No obstante, se ha quedado en el mundo de las intensiones y de los discursos porque no se ha cristalizado completamente en planes, programas y acciones concretas" (González, 1999, p.1).

Hay que estar atenta que estos procesos no estén subyugados a meras politicas gubernamentales que fusionan como "acciones estratégicas de los gobernantes en turno para hacer frente a ciertos problemas de gobierno o de la sociedad, por lo que la participación de la sociedad civil en este ámbito es prácticamente nula" (Campos, 2003, 86).

\section{Sobre el concepto de mujer implícito e explicito}

Una de las responsabilidades mayores que implica participar en la construcción de una política pública para la mujer es la referida al compromiso ético de la definición del concepto de mujer que se construye y al que se le apuesta al interior de dicha política.

La revisión crítica del concepto de mujer construido en el marco del documento de Política De Mujeres Constructoras De Paz Y Desarrollo permite señalar unos énfasis implícitos en los asun- tos de producción y reproducción ligados al SER mujer. Esta mirada crítica nos obliga a preguntarnos sobre las representaciones sociales que subyacen al concepto de mujer y a su pertenencia de género (Jodelet 1976; Rodó 1994; Jiménez, Lucumi \& Reyes 2006). Es posible identificar ciertos roles y estereotipos propios de un discurso con marcadas tendencias argumentativas de hegemonía heterosexual, en un reconocimiento binario de pertenencia de género (masculino femenino) y discursos judeo cristianos (Rodó 1994; Lagarde 1997, 2001; Tenorio 2002; Obando 2006c), base de una representación de la mujer como progenitora y protectora, lo que muchas autoras han denominado discursos marianos de pertenencia de género.

Identificarse con estas apuestas de representación de un ser mujer conlleva a que en la gestión de las propuestas a nivel local se intente amarrar a las sujetos de la política pública de la mujer, a instituciones estereotipadas socialmente y adjudicadas por terceros, como es el caso de la familia. Esta construcción del concepto mujer igual progenitora y protectora, facilita el planteamiento de propuestas como las de el desarrollo de "una Secretaría de la mujer y la familia" planteada y defendida en la Sesión de Participación Ciudadana de Julio 2006, por un sector de representantes del Ente estatal, en la Gobernación del Valle del Cauca. Posición corregida con la presentación y aprobación por parte de la Asamblea del Departamento de la creación de "la Secretaria Equidad de Genero para las 
Mujeres Vallecaucanas" la cual es presentada como uno de los logros en el documento de la VI rendición publica de cuentas Enero 1 de 2004 a Septiembre 20 de 2006, de la Gobernación del Valle de Cauca (Gobernación del Valle del Cauca, Octubre 25, 2006, p.18) y certificada su aprobación como proyecto de Ordenanza No. 274 del 13 de Septiembre del 2006 en sus tres debates, por el Secretario General de la Honorable Asamblea Departamental del Valle del Cauca en Septiembre 30 del 2006 (Gobernación del Valle del Cauca, Octubre 4 de 2006).

Si se procede a naturalizar la definición de Mujer, igual progenitora, igual Madre, igual protectora de la familia, en una idealización de lo femenino igual a lo familiar y lo doméstico (Rodó, 1994), quedan en este proceso de construcción de una política pública planteados varios interrogantes, como son por ejemplo los referidos a:

¿Existe en el concepto de mujer que hace referencia a una naturalización de ella en función de alguna actividad, como es el caso de la reproducción y producción al interior de la familia - un proceso de invisibilidad de la mujer como SUJETO (Fernández 1999; Lamas 1994, Ramirez 2002) de la política pública?

¿Se hace un juego con esta esencialización de la mujer implícita en el discurso de la política pública nacional de la mujer a la invisibilidad histórica (Castellanos 1991; Luna 2004) que se ha construido sobre la presencia reflexiva y creadora de las mujeres en los ámbitos públicos, particularmen- te aquellos referidos a la construcción de conocimiento y política? Si asumimos el reto de construir una política pública de mujer con perspectiva de género amarrada a una política social es necesario admitir que "la política social debe constituirse en una política de fortalecimiento de la ciudadanía social, en la cual la reivindicación de los derechos sociales, la cooperación, la participación y el rescate y fortalecimiento de la esfera pública son elementos constitutivos de su eje nuclear (Velásquez, 1999). Es decir se hace necesario la apropiación y reconocimiento de la política pública de mujer como política social por parte de la ciudadanía. Este proceso pasa por que las mujeres ciudadanas valoren y desarrollen "dispositivos sociales y de presión política que permitan su asimilación y apropiación por parte de la administración local." (González, 1999, p.20). Es este sentido se comparte la opinión de González, cuando plantea que "toda política social debe ser concertada y deben intervenir, de manera activa y propositiva, distintos actores en su formulación, aplicación y monitoreo" (González, 1999, p.5). Es de rescatar el papel protagónico que ha asumido en la actualidad la Confluencia Departamental de Procesos Organizativos de mujeres como Ente representativo de 96 ONGs en el Departamento del Valle del Cauca, anudado a la participación histórica de grupos de mujeres en los desarrollos de políticas públicas que afectan a las mujeres a nivel municipal y departamental, estos protagonismos 
“..han aumentado el ritmo y la cantidad de demandas sociales como consecuencia de la toma de conciencia ciudadana, del fortalecimiento de redes asociativas...." (González, 1999, p.3)

Otro de los interrogantes por resolver se refiere a ¿Cuál es el espacio que le corresponde en esa Política Pública a aquellas mujeres que se definen a sí mismas como mujeres sin hacer énfasis, o una referencia directa a una forma de ser estereotipado como madres, esposas, o hijas? Si partimos del supuesto que la construcción de ese estereotipo obedece a "...un proceso racional, definido por normas socialmente construidas que ordenan las relaciones sociales y vuelven los comportamientos previsibles, aunque esas normas estén o no legalmente formuladas" (Sonia Fleury citada en González, 1999, p.4). Es decir a aquellas mujeres que de manera consciente se proponen existir en su calidad de transgresoras de estereotipos clasistas y machistas de género. En una referencia al quehacer de algunas mujeres políticas ecuatorianas Silvia Vega (2005), señala el "dilema entre representación general y representatividad de género" (Vega, 2005, p. 196-198), como uno de los problemas a los que se enfrentan en las áreas de intervención las mujeres que incursionan en política: Ella plantea como en el imaginario de los varones las áreas de vinculación están referidas de forma limitada a espacios de acción sobre asuntos de niños, familia, y protección mientras en el imaginario de las muje- res puede tratarse de áreas muy diversas, esas que asumen responsabilidades desde asuntos amazónicos hasta aquellos referidos a la gestión pública y de seguridad (Obando, 2006d). En un rescate del papel de las mujeres como ciudadanas participes en la construcción de esta política pública se comparte la opinión de González cuando plantea que como "ciudadanos también tienen responsabilidades y deberes para asumirse como miembros de una colectividad, como actores activos y participativos del desarrollo económico, social, cultural, político de una localidad región o pais" (González, 1999, p.5).

Si se aspira a participar en la construcción de una política pública de mujer con perspectiva de género se hace necesario aclarar qué es lo que une en la diversidad a los sujetos de este grupo. Lo que nos obliga a plantearnos la pregunta sobre ¿Cuál es el lugar para aquellas que se inscriben en una perspectiva de Género que lucha contra la inequidad y la discriminación de las mujeres en sus diversidades de clase, etnia, preferencia sexual, creencias religiosas o posicionamientos políticos, color de piel, etc.? En la opinión de varias autoras se identifica a la exclusión basada en el sexo y amarrado a la apariencia de un cuerpo biológico femenino como una de las experiencias en común más significativa. Esta experiencia de exclusión obligaría a las mujeres a reconocer cuales son sus intereses comunes. (Htung 2005, Obando 2006d). Sin embargo como lo plantea Lourdes Hurtado, atacar las 
causas directas del problema de exclusión de las mujeres tiene implicaciones que superan la mera construcción de una normatividad jurídica en tanto esta exclusión como fenómeno

... es mucho más complejo y tiene que ver con imaginarios sociales, estructuras económicas, identidades, cuestiones étnicas, etc.; por ello, éstas medidas deben ir acompañadas de otro tipo de acciones llevadas a cabo desde el Estado y la sociedad civil que ayuden a atacar la razones estructurales que dan origen al problema. (Hurtado 2005, p.139)

Es así como se hace necesario en el proceso de construcción de una pública de Mujeres con perspectiva de Género el tomar consciencia sobre las diversas formas discursivas de representar el Ser mujer. Aquellas que van desde la representación de mujer madre, cabeza de familia, víctima de maltrato, objeto de agresión y agravio en situaciones de conflicto, en situación de riesgo epidemiológico, objeto de promoción ciudadana, entre muchas otras. Indagar sobre los referentes de estas lecturas del Ser Mujer permitirá el no caer en el desarrollo de ofertas que desempoderan a las mujeres como sujetos que construyen sus propias vidas a pesar de las adversidades. Desafortunadamente en el documento de Política De Mujeres Constructo- ras De Paz Y Desarrollo se recrea a la mujer desde unos planos no continuos, fragmentarios del desarrollo en los cuales predomina la mirada de riesgo, la imagen de una mujer vulnerable, sujeto de protección, en tanto es amenazado constantemente en su condición de sujeto social.

Las dinámicas del conflicto de la mujer, no deben asimilarse exclusivamente con los fenómenos de violencia en los que se ven involucradas como víctimas, también, es necesario reconocer otras fuentes de conflicto como las formas de exclusión que se construyen a nivel económico, político, social, cultural cuya apariencia es menos adversa pero no por eso menos avasalladora.

Una lectura detallada de las áreas programáticas, ejes de la Política De Mujeres Constructoras De Paz Y Desarrollo, como son: el "Empleo y desarrollo empresarial, educación y cultura, salud, salud sexual y reproductiva, violencia contra las mujeres, participación política, comunicaciones, mujer rural, fortalecimiento institucional." (Presidencia de la República, 2005, pp.22-63), señalan cierta discrepancia con el centralismo en el eje temático de la familia que contempla el marco legal (normas constitucionales, leyes, decretos), referido en el documento, fundamento de esta oferta programática diversa. 
Tabla 1. Marco legal referido al tema familia

\begin{tabular}{|l|r|c|c|}
\hline \multicolumn{1}{|c|}{ Marco legal } & Cantidad & $\begin{array}{c}\text { Referidos } \\
\text { a la familia }\end{array}$ & Porcentaje \\
\hline Normas constitucionales & 9 & 5 & $55 \%$ \\
\hline Normas internacionales & 33 & 2 & $6 \%$ \\
\hline Leyes anteriores a la constitución & 36 & 30 & $83 \%$ \\
\hline Leyes posteriores a la constitución & 42 & 15 & $35.7 \%$ \\
\hline Decretos & 12 & 3 & $25 \%$ \\
\hline Total & $\mathbf{1 3 2}$ & $\mathbf{6 0}$ & $\mathbf{2 2 \%}$ \\
\hline
\end{tabular}

Es significativo en la tabla (1) el alto número 60/132 de leyes citadas que hacen referencia al aspecto de familia, de las cuales el 55\% corresponden a las normas constitucionales y un $83 \%$ se refieren a las leyes anteriores a la constitución de 1991, y sirven de sustento a una política de la mujer. Aspectos referidos a la diversidad social, cultural y política de este grupo de mujeres son marginales. Las diferencias de clase, etnia, de territorialidad parecen no tener el mismo grado de significación cuando se construye una política para la mujer y ésta es amarrada a la institución y función familiar. La multiplicidad de intereses, necesidades, formas de resistencia de este grupo específico son ignoradas.

\section{Agentes que participan en la construcción, gestión e implementación de la política pública de la mujer}

El segundo tema de este artículo, está referido a los agentes que participan de la construcción, gestión e implementación de esa política pública de la mujer, él remite por obligato- riedad a la discusión sobre uno de los objetivos y ejes temáticos de la Política De Mujeres Constructoras De Paz Y Desarrollo, la participación.

El concepto de participación política es abordado en América Latina desde la década de los $80^{\circ}$, con un enfoque Psicosocial crítico y una perspectiva epistemológica construccionista. Los estudios de participación recogen los aportes de autores como Tomasatta 1975; Martín Baró 1988; Diaz Bourdenave 1985; Montero 1996; Fernandez Christlieb 1987; Restrepo 2002; Arango 2006, Obando 1992-2006, entre otros, con investigaciones sobre los ámbitos, tendencias, modalidades, procesos y mecanismos de participación. En todos estos trabajos se le reconoce al fenómeno de participación su carácter social, y se le define como un acto social y político de construcción de realidad ejecutado por un sujeto con las mismas características. Asumir la participación en estos términos implica el reconocer a las mujeres como actores sociales y políticos capaces de construir realidad acerca de su propio devenir. Es decir el aceptar que las mujeres poseen un conocimiento concre- 
to sobre su propia realidad y que solamente es posible tener acceso a ese conocimiento, si se trabaja de forma conjunta con ellas. Lo que le debería dar a la construcción de está política el valor de diálogo de sáberes, compromisos y negociaciones entre mujeres (Centro de Estudios de Género, 2006b). Arango (2006), caracteriza el comportamiento participativo como un:

"proceso de dialogo e intercambio colectivo en el cual un conjunto de personas se inscriben en una trama de sentido común orientada a la puesta en juego de unos principios o valores en función de las cuales se acuerden pautas y reglas de comportamiento grupal que orientan la acción transformadora de la realidad y el aumento de la autonomía" (Arango 2006, P. 136).

Promover el comportamiento participativo en la formulación de las políticas públicas de la mujer implicaría el promover el dialogo e intercambio colectivo entre las miembros de un grupo, que dan cuenta de una diversidad en sus formas físicas, cognitivas, emocionales y éticas de ser mujer, pero al mismo tiempo sujetos que comparten intereses y valores como grupo de género, por ejemplo el interés en la emancipación.

A la participación le subyace la posibilidad que posee la población para intervenir en forma activa en la producción de conocimientos relevantes para la generación de cambios dentro de la sociedad y del orden social existente. En este sentido se asume por participación política, el esfuerzo organizado para ejercer y fortalecer el control y seguimiento sobre los recur- sos propios y de las instituciones. Esto debe tener lugar en determinados espacios sociales, y por diferentes grupos y movimientos, los cuales hasta ese momento habían sido aislados en el ejercicio de esa actividad (Obando, 2006a). El compromiso de la política pública de mujeres debe estar orientado a jalonar procesos a través de los cuales se logre transformar y ejercer poder para construir una nueva realidad social acorde a la diversidad de identidades (Butler, 1990), existentes en las formas de ser o posicionarse como mujer. Es una emergencia de nuevas subjetividades políticas potencialmente emancipadoras, ligadas al reclamo de los derechos (Restrepo 2002).

Desde las políticas públicas la participación es entendida como "un proceso social que resulta de la acción intencionada de individuos y grupos en busca de metas específicas, en función de intereses diversos y en el contexto de tramas concretas de relaciones sociales y de poder." (Velásquez \& González, 2003 P.19-20).

Otálvaro $(2005,2007)$, en sus textos de investigación sobre participación política de jóvenes plantea, que la tarea de motivar la participación política en el diseño e implementación de políticas públicas integrales, participativas e incluyentes, sólo es posible, si se construyen sobre la base de principios y estrategias que recurran al conocimiento sistemático de las problemáticas, al diálogo y la mediación como mecanismo de legitimación del Estado y la función pública, al reconocimiento de los 
derechos fundamentales de los ciudadanos y a la promoción de procesos de desarrollo económico local, como factores que permitan mejorar la calidad de vida y el ingreso de los y las sujetos que se encuentran en condiciones de exclusión social y vulnerabilidad. Por tanto como es planteado en el documento borrador de los lineamientos es preciso enfatizar que:

"los alcances de esta politica [la departamental de mujeres del Valle del Cauca] se ubican en la posibilidad de crear condiciones para lograr la igualdad de oportunidades y el ejercicio de los derechos de las mujeres, reconociendo sus diversidades y trazando estrategias para aquellos sectores [de mujeres] que están en situación de mayor desventaja por su pertenencia étnico, cultural, territorial y condición socioeconómica" (Centro de Estudios de Género, 2006b, p.32).

Es decir, se trata de minimizar los riesgos de que en la construcción de esta política pública como lo plantea González, se cumpla formalmente con la creación de canales de participación pero que dichos canales nazcan vacíos de contenido (González, 1999, p.7).

En los procesos de construcción de una política pública para la mujer liderados a nivel local es necesario aclarar ¿cuales son los Agentes que participan en la construcción de esa política pública de la mujer? ¿Cuales son sus intereses, sus dinámicas, sus mecanismos? ¿Cómo lograr que la participación de los diferentes agentes involucrados garantice el tratamiento del componente de género?
Una de las vías identificadas es la de crear situaciones de participación abierta que optimicen los componentes específicos de género (para el caso, referido a lo femenino), en las gestiones que conlleven a la formulación de una política pública de la mujer, con la colaboración de entes gubernamentales y privados, como las ONGs y con las mujeres pertenecientes a las diversas agrupaciones y movimientos de base, afectadas directamente por la política. Es decir, crear las condiciones para un dialogo abierto, una conversación de sáberes sobre el tema. Una situación en la cual cada uno de los involucrados reconozca un espacio para plantear la diversidad de sus intereses, necesidades y valores de género de manera explícita.

\section{Situación actual del desarrollo de la política pública de la mujer a nivel departamental}

Una revisión del estado actual de proceso de elaboración de una propuesta para una política pública de la mujer que se lidera desde la Gobernación del Valle del Cauca en una acuerdo con la Confluencia Departamental de Procesos Organizativos de Mujeres y en convenio con el Centro de Estudios de Género Mujer y Sociedad, de la Universidad del Valle, permite identificar algunas acciones del proceso como relevantes:

Primero las actividades lideradas desde la Gobernación del Valle del Cauca, entre otras es significativo el 
encuentro de 96 organizaciones en base a cuyos sáberes se construye y concerta el Acuerdo Social suscrito entre la Gobernación del Departamento del Valle del Cauca y la Confluencia de Procesos Organizativos de Mujeres. Los ejes priorizados en el proceso de concertación fueron: Trabajo y generación de ingresos, Participación social y política, Salud sexual y reproductiva y erradicación de la violencia contra mujeres.

El encuentro departamental de organizaciones de mujeres en febrero 2006, que trata como tema central la propuesta: creación de la Secretaría de la mujer. Así mismo la Sesión De Participación Ciudadana, de Julio 2006, cuya finalidad fue la de escuchar a las participantes de organizaciones de mujeres, recoger aportes y recomendaciones sobre importancia de la creación de la secretaria de la mujer, con relación a sus funciones, y su impacto. Se reitera allí el papel protagónico de las ONGs desde las cuales como lo plantea González “... han desarrollado nuevas formas de relación entre el Estado y las ONGs, que se materializan en la coordinación, concertación y contratación para formular politicas a nivel local, realizar programas $y$ proyectos y prestar servicios sociales" (González, 1999, p.7). De relevancia es la firma Acuerdo Social entre la Gobernación Departamental y la Confluencia Departamental de Procesos Organizativos de Mujeres ${ }^{5}$ en el contexto del plan de desarrollo 2004 2007 "Vamos juntos por el Valle del Cauca", al interior del cual se desarrolla del Proyecto: "fortalecimiento de la participación social y política de la igualdad de oportunidades para mujeres y hombres". Este proyecto posee dos componentes: la formación política para las mujeres y la construcción de política (Gobernación del Valle del Cauca, 2005). Estos componentes se desarrollan a través de un Convenio entre el Centro de Estudios de Género Mujer y Sociedad, de la Universidad del Valle y la Secretaría de Desarrollo Social de la Gobernación del Valle del Cauca. En analogía a lo que plantea Gonzáléz estas “....ONGs han defendido su papel de agentes de fiscalización y control social frente a la gestión de las administraciones municipales" (González, 1999, p.7).

Uno de los logros del componente de construcción de política de este proyecto es la elaboración del primer borrador del documento "Lineamientos de política de equidad de Género para las mujeres de diferentes sectores y grupos étnicos del Valle del Cauca", asumido por el Centro de Estudios de Género Mujer y sociedad de la Universidad del Valle, en sus lineamientos y líneas de acción se inscribe en el proceso del plan de acción pactado en el 2005 y se basa en las necesidades e intereses mas urgentes planteados por las mujeres de los diferentes municipios como son:

Mas de 27 organizaciones y redes de mujeres del Valle del cauca coordinadas por la secretaria de desarrollo social de la Gobernación. 
"Eje equidad económica, eje equidady territorio, tierra y vivienda, eje equidad en educación y cultura, eje equidad en salud integral, eje vida digna libre de violencias contra las mujeres, eje equidad en acceso a la justicia, eje equidad en participación política (Centro de Estudios de Género, 2006b, p33-34).

Este documento fue entregado en Noviembre del 2006 a un "Comité de conocedoras de la temática" coordinado por la profesora e investigadora, Marta Londoño. Este documento borrador se constituye en la primera fase del proceso, de elaboración de una propuesta más plural, la cual para su construcción requiere de una validación del mismo a través de diversas actividades de socialización, discusión y validación de los contenidos propuestos. Se proyecta su construcción en mezas de trabajo que conglomere mujeres de diferentes organizaciones con su diversidad de intereses a nivel municipal y departamental.

Una tercera actividad esta ligada al componente de formación política para mujeres del proyecto "Fortalecimiento de la participación social y política y promoción de la igualdad de oportunidades para mujeres y hombres, componente construcción de la política". Se constituye en la implementación de una "escuela de formación en género para la incidencia política de las mujeres en los espacios locales" $"$ para integrantes de or- ganizaciones de mujeres en diferentes municipios del Valle del Cauca, Estas actividades de formación se desarrollan en un trabajo de colaboración entre la Gobernación del Valle, el Centro de Estudios de Género, Mujer y Sociedad de la Universidad del Valle. Según el documento propuesta se

"trata de ofrecer un camino lógico de fácil recorrido para las mujeres participantes, que las lleve a incorporar los avances políticos y jurídicos logrados por las mujeres, con una perspectiva histórica, de tal manera que puedan realizar aplicación de tales conocimiento en su contexto cotidiano y local" (Centro de Estudios de Género, 2006a, p.9).

Los temas ofertados son: "¿Y como andamos las mujeres? ¿Cómo llegamos a estar como estamos? ¿Y nuestros derechos hoy? herramientas para la incorporación de la equidad de género en las áreas sectoriales del desarrollo local, Políticas Publicas, escenario de interlocución Mujeres-Estado, Herramientas de Advocacy para el seguimiento y continuidad de las acciones de incidencia" (Centro de Estudios de Género, 2006a, pp.9-11). Uno de los logros que se espera obtener de esta experiencia de formación se refiere a la recolección de textos (escritos y orales) críticos sobre la situación actual y el futuro de las mujeres a partir de los propios aportes de las mujeres de las organizaciones de base participantes. De esta manera se reconoce la existencia de un saber popular en estas parti-

6 Desarrollado en las sedes que tiene la Universidad del Valle en el departamento, espacios que fusionan como punto de encuentro de las mujeres de los distintos municipios. 
cipantes y de este saber como instrumento para la comprensión de la situación de enajenación y marginalidad. La formulación y presentación de propuestas a la administración local para mejorar la condición y la posición de las mujeres y de la comunidad en general es uno de los efectos sociales esperados de este proyecto. Así mismo el fortalecimiento de grupos de mujeres líderes voceros de los intereses de las otras mujeres y de la comunidad ante las instancias gubernamentales.

\section{Bibliografía}

Arango, Carlos (2006). Psicología comunitaria de la convivencia. Programa editorial Universidad del Valle.

Bernal Olarte Angélica (2006) Coyuntura política en Colombia "causas y azares".

Butler, Judith (1990). Gender trouble. Feminism and the subversion of identity. New York: Routledge.

Campos Covarrubias, Guillermo, (2003) la participación ciudadana en la política pública: una reflexión ciudadana. En: Chavéz Carapia, Julia del Carmén: Participación social: retos y perspectivas, (79-93), MÉXICO, Plaza y Valdéz editores.

Castellanos, Gabriela: "Nueva visión de las causas de la jerarquía entre los géneros” En: Castellanos, Gabriela ¿Por qué somos el segundo sexo? Ediciones Universidad del Valle, Cali, 1991. Pág. 63-83.

Castellanos, Gabriela; y Accorsi Simona (1991). Sujetos Femeninos y Masculinos. Centro de Género, Ed. Universidad del Valle.
Centro de Estudios de Género Mujer y Sociedad (2006a). Proyecto: fortalecimiento de la participación social y política y promoción de la igualdad de oportunidades para mujeres y hombres. Convenio Gobernación del ValleSecretaria de Desarrollo Social- Centro de Estudios de Género, Mujer y sociedad. Documento interno.

Centro de Estudios de Género Mujer y Sociedad (2006b) Lineamientos de política de equidad de Género para las mujeres de diferentes sectores y grupos étnicos del Valle del Cauca. Noviembre del 2006, documento borrador.

Colombia, (2005) Proyecto "Proceso de fortalecimiento de los espacios de participación de las mujeres. Confluencia Departamental de Mujeres del Valle del Cauca - Gobernación del Valle UNIFEM, Santiago de Cali.

Colombia, (2004), Acuerdo social suscrito entre la Gobernación del Departamento del Valle del Cauca y la Confluencia de procesos Organizativos de Mujeres, Cali.

Colombia, (Octubre 4, 2006), Certificación de la creación de la Secretaria de Equidad de Género para las mujeres Vallecaucanas. Secretario General de la honorable asamblea departamental. Gobernación del Departamento del Valle del Cauca, Cali.

Colombia, (Octubre 25, 2006), Sexta rendición pública de cuentas. Gobernación del Departamento del Valle del Cauca, Cali.

Díaz Bordenave, Juan (1985) . Participación y Sociedad. Buenos Aires. Ediciones Búsqueda.

De la Espriella Adriana (agosto 2001). ¿Por qué una política para las mujeres en Colombia? Corporación SISMA MUJER con aportes de la confluencia Na- 
cional de redes de mujeres. Bogotá.

Fernández, Ana María (1999). Subjetividad y género. Orden simbólico, ¿Orden político? Zona Erógena, 42, 1-11. Disponible en: http:/www.educ.ar/ educar/superior/biblioteca digital/dis-

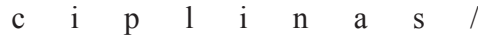
verdocbd.jsp? Documento $=115136$ [accesado: 04.30.2004].

Fernández Christlieb, Pablo (1987). Teoría y método de la psicología política en Latinoamérica. En Marítza Montero (Ed.), Psicología política latinoamericana (pp.69-103). Caracas: Ed. Panapo.

Foucault, Michael (1993). Verdad y poder. En (Editor?) Microfísica del poder (pp.175-189). Madrid: Ediciones de la piqueta.

González R, Esperanza (1999). En busca de la equidad y la convivencia ciudadana. Política social para el municipio de Cali. Ponencia presentada a ISTRLAC. Chile. 1999.

Hurtado, Lourdes (2005). Abriendo puertas: cuotas y participación política de mujeres en el Perú. En León Magdalena (editora), 2005, Nadando contra la corriente mujeres y cuotas politicas en los países andinos. Bogotá. ISBN 95833-7339-7.

Htung, Mala (2005). Democracia e inclusión política: la región andina en perspectiva comparada. En: León Magdalena (editora), 2005, Nadando contra la corriente mujeres y cuotas políticas en los países andinos. Bogotá. ISBN 958-33-7339-7.

Jodelet, Denise (1976). La representación social: fenómenos, conceptos y teorías, 1976.

Jiménez, Mauricio; Lucumi, Eva; Reyes, Aída (2006). Estado de la identidad femenina en adolescentes de 14-18 años. Trabajo de grado para optar al título de psicólogo. Universidad del Valle.

Lagarde, Marcela (1997). "La sexualidad" en los cautiverios de las mujeres: madres, esposas, monjas, putas, presas y locas, México, UNAM, Pág. 177-211. CENDOC-CIDHAL. Disponible en. http:// www.creatividadfeminista.org/ articulos/sex_2003_lagarde.htm. [accesado: 04.30.2004

Lagarde, M. (2001). Identidad Femenina. Disponible en:http:// www.laneta.apc.org/cidhal/lectura/ identidad/texto3.htm\#biblio. [recuperado]:20.02.2004.

Lamas, Marta: Cuerpo: diferencia sexual y género. En debate feminista. Cuerpo y política. Año 5, Vol. 10, Septiembre 1994. Pág. 10-29.

León Magdalena, Holguín Jimena (2005). La cuota sola no basta. En: León Magdalena (Ed.) Nadando contra la corriente. Mujeres y cuotas politicas en los países andinos.

Londoño, Martha Cecilia (2002) El movimiento de mujeres, feminismo y proyecto político en Cali. En Castellanos Gabriela\& Simone Accorsi. (comp), Genero y Sexualidad en Colombia y en Brasil. Manzana de la Discordia Editores. Cali (p.127-172)

Luna, Lola (2004). El sujeto sufragista feminismo y feminidad en Colombia 1930-1957. Ediciones la manzana de la discordia. Cali.

Martín-Baró, Ignacio (1988). Hacia una psicología política latinoamericana. En Gina Pezzano de Vengoechea (Ed.), XIII Congreso Colombiano de psicología. Hacia una psicología para la sociedad Colombiana. Memorias (pp.97117). Barranquilla: Ediciones Uninorte.

Martín-Baró, Ignacio (1994). El método de la psicología política. Textos teoría y 
método. Suplementos Anthropos, 44, 30-40.

Mies, Maria (1984). Methodische Postulate zur Frauenforschung. Beiträge zur feministischen Theorie und Praxis, Frauenforschung oder feministische Forschung?, 11, 7-25.

Obando Salazar, Olga Lucia (1992). Zur Vorschulproblematik in städtischen Armutsgebieten Kolumbiens - das Beispiel des Kinderhausprogramms. Tesis de Maestría, Biblioteca de Ciencias de la Educación, Universidad Técnica de Berlin.

Obando Salazar, Olga Lucia (2002). Antirassistische Bildungsarbeit mit Mädchen - Beiträge $z u$ methodologischen Aspekten der geschlechsts spezifischen Bildungsarbeit mit Mädchen unter Anwendung der Aktionsforschungsmethode. Tesis doctoral (D-82), Biblioteca Ciencias de la Educación, Universidad Técnica de Berlín.

Obando Salazar, Olga Lucia (2005). La capacitación antirracista con perspectiva de género. Un aporte metodológico. Revista la Manzana de la discordia, 1(1), 49-69.

Obando-Salazar, Olga Lucia (2006a, Septiembre). La Investigación Acción Participativa (IAP) en los estudios de psicología política y de género [87 párrafos]. Forum Qualitative Sozialforschung / Forum: Qualitative Social Research [On-line Journal], 7(4), Art. 3. Disponible en: http:// www.qualitative-research.net/fqstexte/4-06/06-4-3-s.htm [fecha de acceso]: 2006, Octubre, 15].

Obando-Salazar, Olga Lucia (2006b). Una experiencia de Investigación e intervención antirracista con perspectiva de género. Revista Interamericana de Psi- cología (RIP). Su Volumen 40, Número 3, pp. 313-332.

Obando Salazar, Olga Lucia (2006c). Informe de investigación proyecto Luna Roja: fortalecimiento de identidad femenina en niñas y jóvenes con experiencia de Maltrato. Instituto de Psicología, Universidad del Valle. Sin publicar.

Obando Salazar, Olga Lucia (2006d). Vale la pena nadar contra la corriente en asuntos de cuotas políticas. Revista $\mathrm{La}$ Manzana de la discordia. ISNN 19007922. Centro de Estudios de Género, mujer y sociedad. Universidad del Valle. En prensa.

Otálvaro, Bairon (2005) "Debates y reflexiones sobre la Política de Juventud en Cali". Revista Comunidad - Centro de Asesoría Territorial CAT. CENCOA. Santiago De Cali.

Otálvaro, Bairon (2007). Análisis de los procesos de participación y organización social para la construcción y evaluación de políticas públicas sociales en el ámbito local. Tesis de Maestría en políticas públicas, Facultad de administración, Universidad del Valle.

Palazón, Francisco (1993). Implicación, acción-reflexión-acción. Revista de Estudios Sociales y de Sociología Aplicada, 92, 43-58.

Presidencia de la República (octubre 2005). Política de mujeres constructoras de paz y desarrollo. Disponible en: http:/ /www.presidencia.gov.co/equidad./documento politica.pdf. [Fecha de acceso: $11 / 11 / 06]$

Ramírez, Martha Cecilia (2002) Do centro a la periferia: os diversos lugares da reproducao nas teorias de género. En: Buarque de Almeida, Heloisa; Gomes costa,Rosely; De Souza Érica Renata (organisadoras). Genero em matizes. Editora da Universidade Sao Francis- 
co. Braganca paulista, Brasil. (p.115152).

Restrepo, Darío (2002). Las prácticas participativas: entre la socialización y la privatización de las políticas públicas, en enlaces y rupturas. Bogotá: Diakonia - Parcomun.

Rodó, Andrea (Septiembre 1994). El cuerpo ausente. En debate feminista. Cuerpo y representación social. Año 5, Vol. 10, 81-94.

Roth Deubel, André-Noel (2004) . Politicas Públicas. Formulación, implementación y evaluación. Bogotá, Ediciones Aurora.

Tomasetta, Leonardo (1975). Participación y Autogestión. Buenos Aires. Amorrortu.

Tenorio, Maria Cristina (2002). Identidad femenina y sexualidad en adolescentes urbanas de estratos 1 y 2 . Colciencias, Universidad del Valle. Centro de investigaciones en psicología, Cali.
Torres Salcido, Gerardo (2003), Políticas públicas y participación social: retos y limitaciones de los programas de combate a la pobreza (un estudio de caso de política2s alimentarias). En: Chavéz Carapia Julia del Carmén: Participación social: retos y perspectivas, (79-93), MÉXICO, Plaza y Valdéz editores. (43-68)

Velásquez, Fabio (Octubre, 1999). Gestión Local y política social. Notas para la definición de un enfoque. Revista Foro, Número 37, Santa Fé de Bogotá.

Velásquez, Fabio \& González, Esperanza (2003). ¿Qué ha pasado con la participación ciudadana en Colombia? Bogota: Ed. Fundación Corona.

Zúñiga, Miryam (1996). Los modelos pedagógicos de algunas experiencias colombianas de educación no formal para el empoderamiento de las mujeres. Revista de la Universidad Popular, 2(2), 38-43. 